- Under specialist practice conditions, resin-modified glass-ionomer cement (RMGIC) restorations are shown to be highly successful.

- Class II RMGICs were as successful as preformed metal crowns.

- Emphasis is placed upon only placing intra-coronal restorations in minimal cavities, preformed crowns being used where the decay process is more advanced.

- High usage of rubber dam reflects the authors' firmly held belief that not only does it provide moisture control, but aids considerably in behaviour management.

\title{
The survival of resin modified glass ionomer and stainless steel crown restorations in primary molars, placed in a specialist paediatric dental practice
}

\author{
J. F. Roberts, ${ }^{1}$ N. Attari ${ }^{2}$ and M. Sherriff ${ }^{3}$
}

Aims To prospectively report on the survival of resin-modified glass ionomer cement (RMGIC), photac-fil and pre-formed stainless steel crown (SSC) restorations in primary molar teeth placed over a seven-year period in a specialist paediatric dental practice under private contract of remuneration.

Method All primary molar restorations placed by a specialist paediatric dentist over a seven-year period were reviewed and the outcome results recorded. Data were recorded at review visits until June 30, 2003. Data recorded included Class I restorations, Class II restorations and SSC. The Class II cavities were either mesial or distal, with or without buccal/palatal extensions. If both proximal surfaces were decayed or if after cavity preparation the resultant outline form was significantly larger than the minimal classical form, RMGIC was not used; an SSC was placed instead. Stainless steel crown preparation followed conventional guidelines. The crowns were cemented with reinforced zinc oxide and eugenol (Kalzinol). The status was recorded as satisfactory restoration, tooth exfoliated, tooth extracted for orthodontic reasons with the date of extraction, or needing replacement. If replaced then the reason for replacement was also recorded.

Results A total of 544 Class I RMGICs, 962 Class II RMGICs, and 1,010 SSCs were placed. At the last review of each restoration, $98.3 \%$ of Class I, 97.3\% of Class II RMGICs and $97.0 \%$ of SSCs were either satisfactory or withdrawn intact.

Conclusion Under the conditions of private specialist practice-based study SSCs continued to prove very successful for the restoration of larger cavities and for pulp-treated primary molar teeth. For the smaller cavities RMGIC were also very successful.

${ }^{1}$ Specialist Paediatric Dental Practitioner, 33 Weymouth Street, London, ${ }^{2}$ Specialist Paediatric Dental Practitioner, 33 Weymouth Street London, ${ }^{3}$ Senior Lecturer, GKT Dental Institute, Dept Dental Biomaterial Science, London

Correspondence to: John Roberts, 33 Weymouth Street, London W1G 7BY

Email:john@paediatric-dentistry.co.uk

\section{Refereed paper}

Received 25.09.03; Accepted 23.03.04

doi: $10.1038 /$ sj.bdj.4812197

๑ British Dental Journal 2005; 198: 427-431

\section{INTRODUCTION}

Levels of dental caries in children in developed countries have been reducing since the early 1970s. However, this decline has now reached a plateau and there is clear evidence that treatment of caries in the primary dentition is still an integral part of child healthcare. ${ }^{1}$

In the last century, different techniques and materials became available for the restoration of primary teeth. In the past, stainless steel crowns (SSCs) have been shown to provide the most durable restorative material for primary molars., ${ }^{2,3}$ However, they have been mainly recommended where pulp therapy has been performed, or in teeth with multi-surface restorations due to developmental defects or caries, or where other restorative materials are likely to fail. ${ }^{4}$

Traditionally, amalgam has been the main restorative material for small Class I and Class II cavities in primary dentition. ${ }^{5}$ In recent years, there have been increasing concerns regarding the use of amalgam because of potential mercury toxicity, and also its effect on the environment, although in a recent review article it was concluded that the mercury released from amalgam restorations was insufficient to cause medical problems. ${ }^{6}$ However these concerns have led to an increasing demand from parents of paediatric dental patients for non-mercury materials. Peretz and Ram ${ }^{7}$ found that although $40 \%$ of parents had no preference for the restorative material for their children, only 12\% preferred amalgam and $48 \%$ preferred tooth-coloured restorations. Parental preference was determined more by health concerns than aesthetics. This demand, together with the many developments which have taken place in restorative dentistry in recent years, have helped encourage increased use of alternative restorative materials. These include composites, glass ionomer cements, resin modified glass ionomer cements and compomers.

Composites, made of part resin and part silanised glass, form a micro-mechanical bond to enamel and dentine. Advantages of composites include good aesthetics, wear-resistance, minimal cavity preparation and ability to reinforce residual tooth structure. ${ }^{8-10}$ 


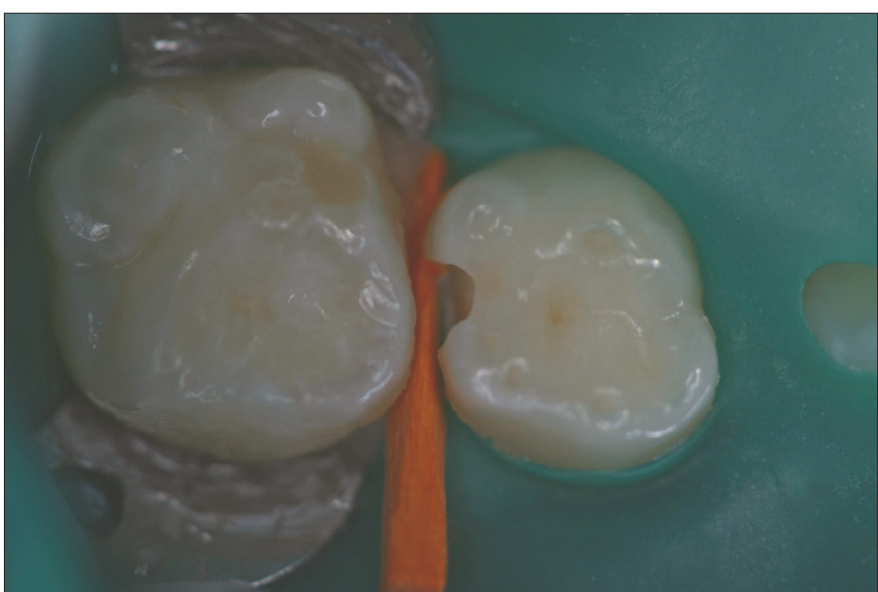

Fig. 1 To show typical outline form for RMGIC Class 2 cavity

However, evaluation of the success rate of posterior composite resin in the primary dentition has shown lower success rates compared to amalgam and other adhesive materials mainly due to micro-leakage, bond failure and recurrent caries. ${ }^{11,12}$

Glass ionomer cement (GIC) has been available for three decades. ${ }^{13}$ Over this period, there have been further developments leading to variants of this material, which include conventional GIC, resin-modified GIC (RMGIC) and compomers. Conventional GIC and its other derivatives all contain fluoroaluminosilicate glass and polyalkenoic acid, which undergo an acid base reaction to form a cement, water being an essential component in this reaction. ${ }^{14}$

Conventional GICs have been used in restoration of primary teeth since their early days of development. The main advantages include their fluoride-releasing property, ${ }^{15}$ and their ability to form a true chemical bond to tooth structure. ${ }^{16,17}$ However, despite possessing reasonable compressive strength, traditional GICs are brittle, with low tensile strength and wear-resistance, which makes them unsuitable for load-bearing restorations in primary teeth. ${ }^{18}$

RMGICs were developed partly to overcome problems of conventional GICs. These cements contain not only the basic component of GICs, but also a monomer such as 2-hydroxyethyl methacrylate (HEMA) or bis-GMA. Addition of the resin component within the glass ionomer formula has not only increased the working time, thus reducing handling difficulties, but it has also substantially increased wear-resistance and physical strength of the cement. ${ }^{19,20}$

There have been very few documented clinical trials reporting the success rate of RMGICs for restoration of primary molars. Croll et al. ${ }^{21}$ reported a 93\% success rate in Class I and Class II restorations after four years. Similarly, Hübel and Mejàre ${ }^{22}$ reported a 94\% success rate in Class II restorations after three years. Mjor et $a l .{ }^{23}$ reported on the longevity and failure of restorations placed in primary teeth in 1996. Of the 2,040 restorations, 314 failed and of these, $30 \%$ were RMGICs and only $17 \%$ were amalgams. The median age of replacement for amalgams was three years, compared to two years for all tooth coloured restorations. However Donly et $a l .{ }^{24}$ found that for Class II cavities RMGICs inhibited caries at the restoration margins better than amalgam.

Roberts and Sherriff ${ }^{2}$ prospectively reported the survival of amalgam and SSC molar restorations, placed in a specialist paediatric dental practice. The true failure rate for primary molar restorations was 4.1\% for Class I amalgams and 11.6\% for Class II amalgams. For SSCs only $1.9 \%$ failed.

It is therefore clear that SSCs are the most reliable and durable restorative material for primary molars. However in cases where there is a minimal Class II cavity, use of SSCs is too invasive and alternative materials need to be considered.
The aim of this present study is to prospectively report on the survival of RMGIC and SSC restorations in primary molar teeth placed over a seven-year period in a specialist paediatric dental practice under private contract of remuneration.

\section{MATERIALS AND METHODS}

All primary molar restorations placed by one operator (JFR) from 1 January 1995 until 31 December 2001 were included in the study. Data were recorded at review visits until 30 June, 2003.

Details recorded and entered onto a computer database were: patient identifying code, birth date, tooth restored, date of placement, restorative material, type of restoration, use of anaesthesia, and use of rubber dam.

Patients were placed on an appropriate review schedule, the usual six to nine month interval being shortened or extended according to individual needs. At that review visit the restoration was examined clinically and radiologically by the same operator (JFR). The study was practice-based, therefore radiographs were taken at varying intervals. Bitewing radiographs would be taken at approximately yearly intervals with a range of six to 24 months, dependent upon the individual patient's risk assessment. The computer database was updated at each review visit with regard to date of re-examination and status. The status was recorded as satisfactory restoration, tooth exfoliated, tooth extracted for orthodontic reasons with the date of extraction, or needing replacement. If replaced then the reason for replacement was also recorded.

It should be noted that not all replacements were due to a failure of the restoration. True failures of intra-coronal restorations would include caries at the margins of or beneath the restoration, fractured restoration, marginal degradation, pulpal infection and partial or total loss of restoration. False failures would be those not directly related to the restoration itself such as caries at a fresh site but which necessitated the replacement of the observed restoration. For stainless steel crowns, true failures would be either crown loss following cement failure or perforation of the occlusal surface as a result of wear, whereas false failures would be due to failure of an associated pulp treatment.

Access to both Class I and Class II cavities was made using highspeed water-cooled tungsten carbide burs, 330 pattern, and then caries removal achieved with low-speed rose-head burs. No extension of outline form was made into sound but caries-susceptible pits and fissures as would have been necessary for amalgam restorations. ${ }^{2}$ Instead, having satisfied the criteria of caries removal, caries prevention was accomplished by applying fissure sealant over both the occlusal RMGIC and the remaining pits and fissures. For Class II caries, a minimal proximal box was cut to give access to the caries, with cavo-surface margins that approximated 90 degrees, and with clearance of contact at the gingival floor. The proximal box therefore differed very little from that of an amalgam cavity (Fig. 1). The potential fluoride leaching property of RMGIC, and its adhesion to tooth surfaces was not used to justify deliberately leaving unsupported enamel, nor of placing cavity margins in positions inaccessible to normal cleaning procedures; those properties merely served to make RMGIC restorations more able to compensate for less-than ideal cavity preparations.

It should be noted that Class II cavities were either mesial or distal, with or without buccal/palatal extensions. If both proximal surfaces were decayed or if after cavity preparation the resultant outline form was significantly larger than the minimal classical form, ${ }^{4}$ RMGIC was not used; an SSC was placed instead.

After thoroughly washing the cavity a matrix band was placed and wedged for Class II cavities. The encapsulated RMGIC (Photacfil) was then mixed and injected into the cavity, with no prior application of primer. The restoration was contoured, excess removed, and exposed to the curing light for 20 seconds. The tooth was then etched for 15-20 seconds, washed for approximately 5 


\begin{tabular}{lccc}
\multicolumn{4}{l}{ Table 1 Use of anaesthesia for RMGIC and SSC restorations } \\
\hline Anaesthesia & Class I & Class II & SSC \\
\hline None & 133 & 42 & 18 \\
& $21.8 \%$ & $3.9 \%$ & $1.6 \%$ \\
Local & 16.5 & -5.0 & -8.9 \\
& 216 & 863 & 485 \\
General & $35.4 \%$ & $79.3 \%$ & $43.8 \%$ \\
& -11.5 & 19.9 & -10.3 \\
& 261 & 183 & 604 \\
Totals & $42.8 \%$ & $16.8 \%$ & $54.6 \%$ \\
& 3.1 & -17.9 & 15.2 \\
In each cell, first number is count, second is percentage, third is adjusted residual. ${ }^{25}$ \\
Pearson $\chi^{2}(4)=652.7776$ \\
Likelihood-ratio $\chi^{2}(4)=620.0433 \quad \mathrm{Pr}=0.001$
\end{tabular}

\begin{tabular}{|c|c|c|c|}
\hline Rubber dam & Class I & Class II & SSC \\
\hline Yes & 468 (76.7\%) & 1,041 (95.7\%) & $1,085(98.0 \%)$ \\
\hline No & $142(23.3 \%)$ & $47(4.3 \%)$ & $22(2.0 \%)$ \\
\hline Totals & 610 & 1,088 & 1,107 \\
\hline
\end{tabular}

Table 3 Ages (years) at which RMGIC and SSC restorations placed

\begin{tabular}{lccc}
\hline Restoration & Maximum age placed & Minimum age placed & Mean age placed \\
\hline Class 1 & 12.64 & 1.95 & 5.83 \\
Class 2 & 15.41 & 2.73 & 7.48 \\
SSC & 15.41 & 1.96 & 6.29
\end{tabular}

Table 4 Review times (years) for RMGIC and SSC restorations

\begin{tabular}{lcc}
\hline Restoration & Maximum time reviewed & Mean time reviewed \\
\hline Class 1 & 7.68 & 2.13 \\
Class 2 & 6.73 & 1.72 \\
SSC & 7.69 & 2.13
\end{tabular}

Table 5 Status of RMGIC and SSC restorations placed, showing all replacements

\begin{tabular}{lccc}
\hline Status & Class I & Class II & SSC \\
\hline Not reviewed & $66(10.8 \%)$ & $126(11.6 \%)$ & $97(8.9 \%)$ \\
Satisfactory & $371(60.8 \%)$ & $664(61.0 \%)$ & $716(64.7 \%)$ \\
Exfoliated & $93(15.3 \%)$ & $241(22.2 \%)$ & $255(23.0 \%)$ \\
Ortho. extraction & $2(0.3 \%)$ & $6(0.6 \%)$ & $9(0.8 \%)$ \\
Replaced & $78(12.8 \%)$ & $51(4.7 \%)$ & $30(2.7 \%)$ \\
Totals & 610 & 1,088 & 1,107
\end{tabular}

\begin{tabular}{lccc}
\multicolumn{4}{l}{ Table 6 Reasons for replacement of RMGIC and SSC restorations } \\
\hline Replacement reason & Class I & Class II & SSC \\
\hline Fresh site caries & 69 & 25 & 0 \\
Recurrent caries & 4 & 12 & 0 \\
Fractured restoration & 0 & 2 & 0 \\
Marginal failure & 5 & 11 & 0 \\
Pulpal infection & 0 & 1 & 0 \\
Lost crown & 0 & 0 & 9 \\
Hole in crown & 0 & 0 & 21 \\
Totals & 78 & 51 & 30
\end{tabular}

Table 7 Status of RMGIC and SSC restorations reviewed after placement, showing mean time to failure

\begin{tabular}{lcccrc}
\hline Restoration & $\begin{array}{l}\text { Satisfactory at } \\
\text { last review }\end{array}$ & $\begin{array}{l}\text { Withdrawn } \\
\text { intact }\end{array}$ & $\begin{array}{l}\text { True } \\
\text { failure }\end{array}$ & Totals & $\begin{array}{l}\text { Mean time } \\
\text { to failure (years) }\end{array}$ \\
\hline Class I & $371(68.2 \%)$ & $164(30.1 \%)$ & $9(1.7 \%)$ & 544 & 2.40 \\
Class II & $664(69.0 \%)$ & $272(28.3 \%)$ & $26(2.7 \%)$ & 962 & 1.78 \\
SSC & $716(70.9 \%)$ & $264(26.1 \%)$ & $30(3.0 \%)$ & 1,010 & 2.48
\end{tabular}

seconds and thoroughly dried. Fissure sealant (Delton) was applied over the RMGIC and any remaining pits and fissures before again being light cured for 20 seconds. Any excess fissure sealant flash was removed after matrix band removal. In almost all cases the occlusion was not checked and no occlusal adjustment was made, the fissure sealant covering to the restoration was instead allowed to self-adjust during normal masticatory function.
Stainless steel crown preparation followed conventional guidelines. ${ }^{4}$ The crowns were cemented with reinforced zinc oxide and eugenol (Kalzinol).

\section{RESULTS}

The distribution of restorations was 610 Class I RMGICs, 1,088 Class II RMGICs, and 1,107 SSCs. The use of anaesthesia is shown in Table 1, where significantly more Class I restorations were placed without anaesthetic than Class II or SSC restorations, and significantly fewer Class II restorations were placed under a general anaesthetic than Class I or SSC.

Rubber dam was used in 76.7\% of Class I, 95.7\% of Class II and 98.0\% of SSC restorations (Table 2 ).

Table 3 shows the maximum, minimum and mean ages, in years, at which the restorations were placed.

The maximum and mean follow-up periods, in years, were 7.68, 2.13 for Class I; 6.73, 1.72 for Class II; 7.69, 2.13 for SSC restorations (Table 4).

Table 5 shows the status of all restorations placed. Restorations where the patient had not returned after placement comprised 10.8\% Class I, 11.6\% Class II, and 8.9\% SSCs. Restorations judged to be satisfactory at their last review included $60.8 \%$ Class I RMGICs, 61.0\% Class II RMGICs and 64.7\% SSCs. Exfoliations accounted for 15.3\% Class I RMGICs, 22.2\% Class II RMGICs, and 23.0\% SSCs, and these were classified as 'withdrawn intact', as were the small number of restored teeth that needed extraction for orthodontic purposes. Replacements were needed for 12.8\% Class I RMGICs, 4.8\% Class II RMGICs and 2.7\% SSCs.

Table 6 shows the reason for replacement of the restorations. All of the replacements for SSCs were true failures, but in the RMGIC groups false failures occurred when caries developed at a fresh site, eg new proximal caries where an occlusal restoration had been placed. Thus 69 false failures for Class I and 25 for Class II restorations were added to the respective 'withdrawn intact' groups, leading to the revised status of restorations shown in Table 7.

Excluding those restorations which had not been reviewed following placement, the true failure rates were as follows; $1.7 \%$ of 544 Class I RMGICs, 2.7\% of 962 Class II RMGICs, and 3.0\% of 1,010 SSCs. The mean times to failure (years) were 2.40 for Class I, 1.78 for Class II, and 2.48 for SSC restorations (Table 7). The Kaplan-Meier survival graph (Fig. 2) shows no significant differences in these survival rates, the probability associated with the Log-Rank test $=0.07$.

\section{DISCUSSION}

The success rates of SSC and RMGIC restorations for primary molar Class I and II cavities under specialist practice conditions have been shown here. However, it should be emphasised that for Class II restorations RMGIC was only used for minimal sized cavities. For larger cavities, or where the decay process involved both proximal surfaces, SSCs were used, the high success rates being consistent with a previously published study. ${ }^{2}$

The frequent use of rubber dam may be a contributory factor to the high success rate of the restorations in this study, not only for maintaining a dry field of operation, but also for its contribution to behaviour management of the child patients.

The results show that a significantly higher percentage of Class I restorations were placed without the use of anaesthesia. ${ }^{25}$ This is attributable to the fact that there is no clear clinical dividing line between a cavity designated as a preventive resin restoration (PRR) where anaesthesia would not be routinely provided, and one planned to be a Class I cavity where anaesthesia would be administered pre-operatively. Not uncommonly in this study, a planned PRR evolved into a larger restoration sometimes involving dentine, but for which patient tolerance permitted completion without anaesthesia. The operator made a clinical 


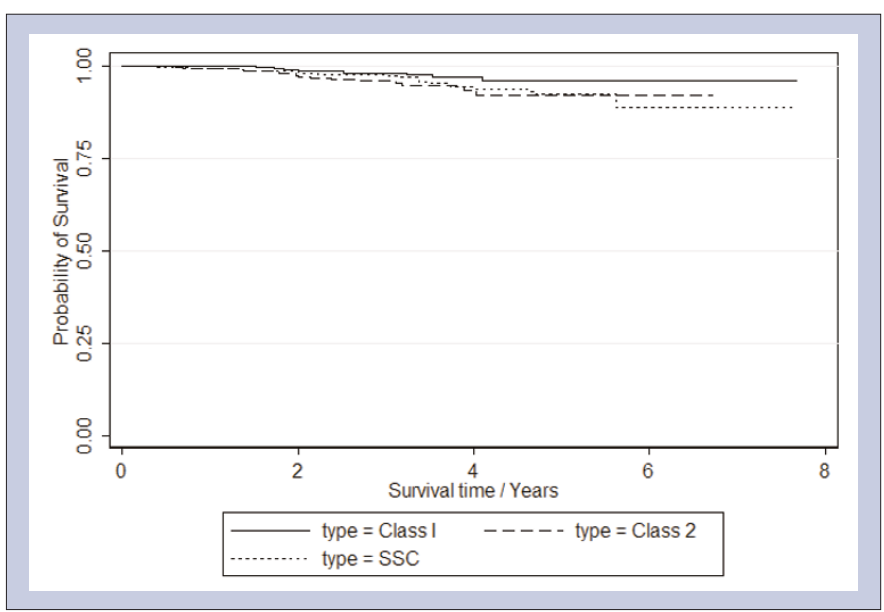

Fig. 2 Kaplan-Meier chart showing survival of restorations

decision at the time of cavity preparation whether to record for the purposes of this study a PRR or Class I cavity depending upon dentine involvement and the percentage of the pit and fissure system involved. This also partly explains why the majority of cases in which rubber dam was not used was for Class I restorations, as the absence of local anaesthesia makes rubber dam unacceptable to many child patients. No anaesthesia was used for 42 Class II RMGICs. Typically these were cases where the adjacent primary molar had been lost and direct access to the proximal surface was possible; often a small cavity could be prepared without anaesthesia. Replacement of SSCs could in most cases be achieved without the use of anaesthesia, as demonstrated by the 18 cases shown in Table 1 .

No cavity liner was used for RMGIC restorations in this study. Murray ${ }^{26}$ showed that the floor of the cavity preparation could approach to within $0.25 \mathrm{~mm}$ of the pulp without causing significant damage to the pulp. The remaining dentine thickness was the most important variable in determining pulpal response to both cutting procedures and the restorative materials, composites, RMGICs, zinc-oxide/eugenol and calcium hydroxide used in their study. Carious dentine has been described as having two distinct zones; the outer superficial layer which is irreversibly demineralised, and the inner layer which has been reversibly involved in the carious process but which still retains the potential to be repaired under suitable conditions. ${ }^{27}$ In view of this and also the fact that in primary molars the enamel and dentine are both relatively thin, ${ }^{28}$ it would be logical to remove the outer zone of the dentine, maintaining the inner zone in order to avoid exposure. In addition to this, more recent research has demonstrated that if deep lesions close to the pulp are treated with antiseptic lining materials such as calcium hydroxide, or cariostatic restorative materials such as GICs, then any residual bacteria retained beneath the restoration would be very unlikely to cause further caries. ${ }^{29-31}$

Although the fluoride-release potential of GICs has often been cited as a reason to use this material and its derivatives for the restoration of primary teeth, it should be noted that the amount of fluoride released varies between brands. ${ }^{32}$

In the present study, fissure sealant was applied after etching, to both the remaining pits and fissures and also to the set surface of the RMGIC. Sidhu and Sherriff, ${ }^{33}$ and Ribeiro ${ }^{34}$ showed that the susceptibility of GIC material to dehydration had not been significantly reduced by the addition of resin in RMGIC, and suggested that surface protection helps with water balance. Chuang et $a .^{35}$ concluded that RMGICs remain moisture-sensitive, and all the samples in their study showed incomplete sealing of the cavity margin. They suggested that resin adhesive should be used to reduce marginal leakage of RMGICs. Mazzaoui et al. ${ }^{36}$ showed that a thin layer of dentine bonding adhesive covering significantly decreased the amount of fluoride released from both GIC and RMGIC. It did not however block it totally and fluoride continued to be released from the RMGIC through the layer of resin. In the present study, resin was not applied to the proximal surface of the Class II restorations, so that there was no inhibition of potential fluoride release against the adjacent tooth surface.

In a previous study the survival was reported of amalgam and SSCs in paediatric dentistry. One operator performed all the restorations and it was emphasised that amalgam was only used for minimal, classical cavities. More extensive carious lesions were restored with SSCs. ${ }^{2}$ In the present study, the same operator again placed all the restorations, thus giving a constant approach to clinical decision-making. The clinical impression gained during the course of this study was that RMGIC was being used in more extensive cavities than would have been the case with amalgam restorations, and that fewer SSCs were therefore employed. However the proportion of Class II amalgam:SSC and Class 2 RMGIC:SSC shows no statistical difference, $\mathrm{p}=0.35$.

It should be noted that not all replacements were due to a failure of the restoration. True failures of intra-coronal restorations would include caries at the margins of or beneath the restoration, fractured restoration, marginal degradation, pulpal infection and partial or total loss of restoration. False failures would be those not directly related to the restoration itself such as caries at a fresh site but which necessitated the replacement of the observed restoration. For stainless steel crowns, true failures would be either crown loss following cement failure or perforation of the occlusal surface as a result of wear, whereas false failures would be due to failure of an associated pulp treatment. With regard to true failure, 30 SSCs failed out of 1,010 reviewed (3.0\%). Cement failure accounted for nine of those and the occlusal surface was worn through in 21 cases. Considering the cuspal wear that naturally occurs in primary molars at age 8-9, it is not surprising that a number of SSCs were also worn through. It is perhaps debatable whether the worn surface of an SSC is a true failure of the crown rather than normal wear and tear. However, if left then gross caries can quickly become established in the dentine beneath the SSC. Therefore in this study the worn-through SSC was replaced preventatively and marked as a failure. Within the RMGIC true failure group, 1.7\% for Class I and 2.7\% for Class II, only one tooth showed evidence of pulpal infection, and only two restorations fractured, all three of these being Class II restorations. The rest were due to marginal degradation; in some cases recurrent caries had started, in others the defect was noted and the restoration replaced preventatively as discussed above with regard to worn-through SSCs. This latter group may not have been considered by some operators to warrant replacement, as the tooth could conceivably have progressed to exfoliation without further breakdown; in this prospective study the operator/assessor was at pains to avoid a favourable bias in the judgement of acceptable restorations. One criterion for the assessment by the operator in this study of a satisfactory restoration was whether any peer-review would also judge it to be satisfactory, knowing it had been placed at a specialist paediatric dental practice. Hence an overpessimistic ruling was made for failed restorations.

\section{SUMMARY}

Under the conditions of this specialist practice-based study of primary molar restorations, SSCs continued to prove very successful for the restoration of larger cavities and for pulp-treated teeth, with $97.0 \%$ satisfactory or withdrawn intact at the last review. For the smaller cavities RMGIC shows promising survival rates with 98.3\% of Class I and 97.3\% of Class II restorations being satisfactory or withdrawn intact at last review. 
1. Murray J. Prevalence of dental caries: retrospect and prospect. Dent Update 1998; 25: 374-378.

2. Roberts J F, Sherriff M. The fate and survival of amalgam and preformed crown restorations placed in a paediatric dental practice. Br Dent J 1990; 169: 237-244.

3. Randall R C, Vrijhoef M M A. Efficacy of preformed metal crowns vs. amalgam restorations in primary molars. J Am Dent Assoc 2000; 131: 337-343.

4. Curzon M E J, Roberts J F, Kennedy D. Kennedy's paediatric operative dentistry. 4th ed. Oxford: Butterworth-Heinemann, 1996. pp 79-87, 114-127.

5. Black G V. Operative Dentistry. Vol 2 - treatment of caries. Chicago: Medico-Dental Pub. Co, 1936. pp 78-112.

6. Osborne J W, Albino J E. Psychological and medical effects of mercury intake from dental amalgam: a status report from the American Journal of Dentistry. Am J Dent 1999; 12: 151-156.

7. Peretz B, Ram D. Restorative material for children's teeth: preferences of parents and children. J Dent Child 2002; 69: 243-248.

8. Nelson G V, Osborne J W, Gale E N, Norman R D, Phillips R W. A three year clinical evaluation of composite resin and a high copper amalgam in posterior primary teeth. J Dent Child 1980; 47: 414-418.

9. Oldenburg T R, Vann W F, Dilley D C. Composite restorations for primary molars: results after four years. Pediatr Dent 1987; 9: 136-143.

10. Vann W F, Burkmeier W W, Mahler D B. Assessing composite resin wear in primary molars: four-year clinical findings. J Dent Res 1988; 67: 876-879.

11. Kilpatrick N M. Durability of restorations in primary molars. J Dent 1993; 21:67-73.

12. Papathanassiou A G, Curzon M E J, Fairpo C G. The influence of restorative material on the survival rate of restorations in primary molars. Pediatric Dent 1994; 16 282-288.

13. Wilson $A$ D, Kent B E. A new translucent cement for dentistry. The glass ionomer cement. BrDent J 1972; 132: 133-135.

14. Mjor I A, Moorhead J E. Selection of restorative materials; reasons for replacement and longevity of restorations in Florida. J Am Coll Dent 1998; 45: 27-33.

15. de Araujo F B, Garcia-Godoy F, Cury J A, Conceicao E N. Fluoride release from fluoride-containing materials. Operative Dent 1996; 21 : 185-190.

16. Mount G J. Clinical placement of modern glass-ionomer cements. Quintessence Int 1993; 2: 99-107.

17. Mount G J. Glass ionomer cements and future research. Am J Dent 1994; 7: 286-292.

18. Combe E C, Burke F J J, Douglas W H. Dental biomaterials. London: Kluwer, Academic Publishers, 1999. pp221-231.

19. Mitra S B, Kedrowski B L. Long-term mechanical properties of glass ionomers. Dent Materials 1994; 10: 78-82
20. Douglas W H, Lin C P. Strength of the new systems. In Hunt P R. (ed) The nex generation (Proceedings of the second International symposium on glass ionomers). Philadelphia: International Symposia in Dentistry, PC, 1994. 209-216.

21. Croll T P, Bar-Zion Y, Segura A, Donly K J. Clinical performance of resin-modified glass ionomer restorations in primary teeth; a retrospective evaluation. J Am Dent Assoc 2001: 132: 1110-1116.

22. Hübel $\mathrm{S}$, Mejàre I. Conventional versus resin-modified glass ionomer cement for Class 2 restorations in primary molars. A three year clinical study. Int J Paed Dent 2003; 13:2-8.

23. Mjor I A, Dahl J E, Moorhead J E. Placement and replacement of restorations in primary teeth. Acta Odontol Scand 2002; 60: 25-28.

24. Donly K J, Segura A, Kanellis M, Erickson R L. Clinical performance and caries inhibition of resin-modified glass ionomer cement and amalgam restorations. J Am Dent Assoc 1999; 130: 1459-1466.

25. Agresti A. An introduction to categorical data analysis. Wiley Series in Probability and Statistics. Chichester: Wiley-Interscience.

26. Murray P E, About I, Lumley P J, Franquin J C, Remusat M, Smith A J. Cavity remaining thickness and pulpal activity. Am J Dent 2002; 15: 41-46

27. Fusayama T. Two layers of carious dentine: diagnosis and treatment. Operative Dent 1979: 4: 63-70.

28. Finn S B. Clinical Pedodontics. 2nd ed. London: Saunders, 1962

29. Shovelton D J. Studies of dentine and pulp in deep caries. Int Dent J 1970; 20: 283-296

30. Bjorndale L, Larsen T, Thylstrup A. A clinical and microbiological study of deep carious lesions during stepwise excavation using long treatment intervals. Caries Res 1997; 31: 411-417.

31. Mertz-Fairhurst E J, Curtis T W, Ergle J W, Rueggeberg F A, Adair S M. Ultra conservative and cariostatic sealed restorations; results at year ten. J Am Dent Assoc 1998; 129: 55-66.

32. Kan K C, Messer L B. Variability in cytotoxicity and fluoride release of resin-modified glass-ionomer cements. J Dent Res 1997; 76: 1502-1507.

33. Sidhu S K, Sherriff M, Watson TF. The effects of maturity and dehydration shrinkage on resin-modified glass-ionomer restorations. J Dent Res 1997; 76: 1495-1501.

34. Ribeiro A P G, Serra M C et al. Effectiveness of surface protection for resin-modified glass-ionomer materials. Quintessence Int 1999; 30: 427-431.

35. Chuang $S F$, Jin $Y T$, Wong $T Y$. Effect of various surface protections on the margin microleakage of resin-modified glass ionomer cements. J Prosthet Dent 2001; 86 309-314.

36. Mazzaoui S A, Burrow M F, Tyas M J. Fluoride release from glass ionomer cements and resin composites coated with a dentin adhesive. Dent Materials 2000; 16: 166-171. 